Bryn Mawr College

Scholarship, Research, and Creative Work at Bryn Mawr College

Graduate School of Social Work and Social

Graduate School of Social Work and Social

Research Faculty Research and Scholarship

Research

2011

\title{
Misbehaviors of Front-Line Research Personnel and the Integrity of Community-Based Research
}

Gala true

Leslie B. Alexander

Bryn Mawr College, lalexand@brynmawr.edu

Kenneth A. Richman

Let us know how access to this document benefits you.

Follow this and additional works at: http://repository.brynmawr.edu/gsswsr_pubs

Part of the Social Work Commons

\section{Custom Citation}

True, Gala, Leslie B. Alexander, and Kenneth A. Richman. "Misbehaviors of Front-Line Research Personnel and the Integrity of Community-Based Research." Journal of Empirical Research on Human Research Ethics 6 (2011): 3-12.

This paper is posted at Scholarship, Research, and Creative Work at Bryn Mawr College. http://repository.brynmawr.edu/gsswsr_pubs/12

For more information, please contact repository@brynmawr.edu. 


\section{Misbehaviors of Front-Line Research Personnel and THE INTEgRity OF COMMUNity-BASEd Research}

Gala True

Philadelphia Veterans Affairs Medical Center

Leslie B. Alexander

Bryn Mawr College

Kenneth A. Richman

Massachusetts College of Pharmacy and Health Sciences

ABSTRACT: THERE HAS BEEN LITTLE EMPIRICAL research into misconduct and misbehavior among community research workers who recruit and collect data in vulnerable and marginalized health populations and are also members of those same communities. We conducted qualitative interviews with community research workers and traditional research assistants to understand the context and consequences of misbehaviors that pose a threat to research ethics and data integrity. In our sample, more community research workers acknowledged engaging in research wrongdoing than did traditional research assistants. These behaviors were most prevalent among community research workers who were not well-integrated into the research team. We suggest best practices for investigators to promote an environment that supports research integrity in research projects that employ community research workers.

KEY WORDS: research integrity, research misconduct, community-based research, front-line research personnel, vulnerable populations, qualitative research methods

Received: July 15, 2010; revised: February 11, 2011

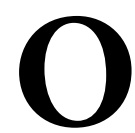
VER THE PAST $3 O$ YEARS, SCHOLARLY INTEREST in research integrity has grown steadily. An initial emphasis on research misconduct, typically defined as "fabrication, falsification, or plagiarism" (FFP), has been broadened to include consideration of "normal misbehaviors" in research (de Vries, Anderson, \& Martinson, 2006). These include ignoring or circumventing human subjects requirements, engaging in questionable relationships with research subjects, and changing the design, methodology, or results of a study
(Anderson, Horn et al., 2007; Anderson, Martinson, \& de Vries, 2007; Anderson, Ronning et al., 2007; de Vries et al., 2006; Martinson et al., 2006; Martinson, Anderson, \& de Vries, 2005).

While efforts to ensure research integrity have broadened the scope of research wrongdoing, attention has remained fairly narrowly focused on scientists and principal investigators as the persons who might engage in wrongdoing. There has been relatively little empirical research into research misbehavior among front-line research personnel. Our qualitative study offers a preliminary look at misbehaviors, including FFP, of street-level research staff called "community research workers" (CRWs).

CRWs are distinguished from other types of research personnel by their shared community with the research population, with community being defined as "a group of people with diverse characteristics who are linked by social ties, share common perspectives, and engage in joint action in geographical locations or settings" (MacQueen et al., 2001). Involvement of CRWs in community-based and translational research has occurred as lay health workers have moved into research either through purposeful efforts to enhance investigators' access to study populations (Hill, Bone, \& Butz, 1996; Perez \& Martinez, 2008) or on an ad hoc basis when a service organization collects data to evaluate effectiveness of programs or contracts with an outside research investigator to facilitate access to study participants. Employing community members who are familiar with the sociocultural and environmental context of the target research population can lead to greater success in study recruitment and follow-up. However, it may also raise new challenges to research integrity.

In previous work, two of the authors, Alexander and Richman, explored through focus groups how CRWs differ from traditional research assistants (Alexander \& Richman, 2008). They found that CRWs tended not to distinguish between ethical challenges and the practical challenges of day-to-day recruitment and data gathering, and had an inclination to reject strict rules when conducting their research activities.

In this paper, we report on behaviors of CRWs that pose a threat to research integrity, describe the contexts in which these actions occur, and explore differences

Journal of Empirical Research on Human Research Ethics, PP. 3-12. PRINT ISSN 1556-2646, ONLINE ISSN 1556-2654. (C) 2011 BY JOAN SIEB ER. ALL RIGHTS RESERVED. PLEASE DIRECT ALL REQUESTS FOR PERMISSIONS TO PHOTOCOPY OR REPRODUCE ARTICLE CONTENT THROUGH THE UNIVERSITY OF CALIFORNIA PRESS'S RIGHTS AND PERMISSIONS WEBSITE, HTTP://WWW.UCPRESSJOURNALS.COM/REPRINTINFO.ASP. DOI: $10.1525 /$ jer.2011.6.2.3 
between CRWs and traditional research assistants in terms of the stressors they experienced and their responses.

Our work builds on that of Martinson, Anderson, de Vries, and colleagues, who demonstrated that the association between perceived distributive injustice (e.g., lack of recognition or reward for one's efforts) and research misbehavior was greater among early-career scientists compared with more senior scientists (Anderson, Martinson et al., 2007; Anderson, Ronning et al., 2007; de Vries et al., 2006; Martinson et al., 2006). Thus, the perception of unfairness in reward of one's efforts was more likely to lead to misbehavior for researchers whose livelihood and/or identity as a scientist were less wellestablished. These findings are relevant to our understanding of misbehavior among CRWs. We argue that CRWs may perceive procedural injustice in the research enterprise itself (e.g., monies being spent to fund research rather than programs in poor communities, the potential stigmatization of the community based on research findings), which may in turn contribute to misbehaviors in research. In addition, CRWs may be more likely to engage in misbehavior when they perceive distributive injustice, compared with traditional research assistants; like early-career scientists, many CRWs identify strongly with their work roles but have more limited career options and resources.

\section{Method}

Participants included three different types of research workers: (a) single-role CRWs, defined as CRWs who are hired solely to carry out research activities; (b) dualrole CRWs, who are employed in service activities in addition to recruiting participants or collecting research data; and (c) traditional research assistants, defined as research personnel who conduct the same research activities with similar populations as CRWs but do not share common characteristics with research subjects (Alexander \& Richman, 2008).

Participants were recruited from nonprofit health and human service and academically affiliated research organizations. Potential participants initiated contact with a member of the study team and were screened for eligibility over the phone. Eligibility criteria for all participants included: Over age 18, fluent in spoken English, and had conducted recruitment, enrollment, or data collection activities on a community-based research study in the past year. Our study was reviewed and approved by the Institutional Review Board of Bryn Mawr College. In addition, we obtained a Certificate of Confidentiality from the National Institute of Nursing Research.

\section{Qualitative Interviews}

A qualitative research methodology was deemed most appropriate for eliciting the self-reported actions of this understudied group of research workers and exploring differences and similarities between the different cohorts (e.g., single-role and dual-role CRWs and traditional research assistants). We decided on a semi-structured interview format to allow participants the freedom to respond according to language and concepts meaningful to them, while keeping the focus on ethical challenges faced in daily research work and actions taken to resolve those challenges (Brand, 2002). The interview guide is described in full detail in the supplementary online document (SOD), which also includes details on how the data were analyzed.

We interviewed 46 front-line research workers: 17 single-role CRWs, 15 dual-role CRWs, and 14 traditional research assistants. A majority of respondents were female (41 out of 46). Traditional research assistants were younger, more likely to be non-Hispanic whites, and had completed more years of education compared with CRWs. Dual-role CRWs had been working in their current research position longer when compared to singlerole research workers and traditional research assistants. Table 1 presents a description of the study sample.

\section{Results}

Our interviews revealed important differences between CRWs and traditional research assistants. Specifically, more dual-role and single-role CRWs said they were aware of misbehaviors or misconduct in research (actions committed by themselves or a colleague) compared to traditional research assistants, and the types of misbehaviors/misconduct the CRWs said they were aware of appeared to be linked to challenges specific to their job functions. Contextual factors played a key role in understanding why some CRWs said they themselves had engaged in research misconduct or misbehavior while conducting research in the community. Analysis of community research worker interviews uncovered a core tension, where the shared characteristics and community that enabled CRWs to successfully conduct research in marginalized health populations also exposed them to greater risk and harm as compared to traditional research assistants. Although faced with the same pressures and challenges, a majority of all respondents said they avoided research misconduct and misbehaviors; our analysis identified protective factors, including personal coping mechanisms and organizational factors. 
TABLE 1. Description of Sample.

\begin{tabular}{|c|c|c|c|c|}
\hline & $\begin{array}{l}\text { Single-role } \\
\text { community } \\
\text { research } \\
\text { worker } \\
n=17\end{array}$ & $\begin{array}{l}\text { Dual-role } \\
\text { community } \\
\text { research } \\
\text { worker } \\
n=15\end{array}$ & $\begin{array}{c}\text { Traditional } \\
\text { research } \\
\text { assistant } \\
n=14\end{array}$ & $\begin{array}{l}\text { Total } \\
n=46\end{array}$ \\
\hline Mean age (SD) & $47(11)$ & $37(10)$ & $29(12)$ & $39(13)$ \\
\hline \# Female & 14 & 13 & 14 & 41 \\
\hline Mean years in this position (SD) & $3.8(3.2)$ & $5.6(5.3)$ & $1.9(1.4)$ & $3.8(3.9)$ \\
\hline \multicolumn{5}{|l|}{ Ethnicity } \\
\hline \# Hispanic/Latino & 3 & 5 & 0 & 8 \\
\hline \# Non-Hispanic/Latino & 12 & 10 & 14 & 36 \\
\hline \# Missing/Refused & 2 & 0 & 0 & 2 \\
\hline \multicolumn{5}{|l|}{ Race } \\
\hline \#African American & 10 & 11 & 4 & 25 \\
\hline \# White & 2 & 0 & 10 & 12 \\
\hline \# Asian & 2 & 0 & 0 & 2 \\
\hline \# Native American & 1 & 0 & 0 & 1 \\
\hline \# Other (Hispanic) & 2 & 4 & 0 & 6 \\
\hline \multicolumn{5}{|l|}{ Education } \\
\hline \# with HS/GED & 4 & 0 & 0 & 4 \\
\hline \# with some college & 10 & 6 & 0 & 16 \\
\hline \# with college degree & 2 & 9 & 11 & 22 \\
\hline \# with advanced degree & 1 & 0 & 3 & 4 \\
\hline
\end{tabular}

\section{Types of Misconduct and Misbehaviors of Front-Line Research Personnel}

Discussion of research misconduct and misbehavior arose throughout the interviews, particularly when respondents talked about how they dealt with ethical challenges they encountered in conducting research activities. Nearly half of all CRWs (15 out of 32) said they had engaged in research misconduct or misbehavior while conducting research activities, while no traditional research assistants said they had engaged in misconduct or misbehavior. Most of the CRWs (27 of 32) said they had personal knowledge of instances where a research worker colleague committed actions that constitute research misconduct or misbehavior. In contrast, slightly less than one-third (4 out of 14) of the traditional research assistants said they had heard of other research personnel fabricating data; these were reports of second- and third-hand knowledge (e.g., events that occurred before they came to work on a study and which were used as examples of "what not to do" during training).

One-quarter of CRWs (8 out of 32) said they had engaged in behaviors that fit the traditional definition of research misconduct (falsification or fabrication of data), while about half ( 15 of 32) had personal knowledge of a colleague who had engaged in such behaviors. Falsification or fabrication of data included instances where a CRW said they either had filled out an entire instrument or measurement on behalf of an actual participant while the participant was not present, or had falsely indicated a participant was present for part of a research intervention. For example, a dual-role CRW talked about how she and her colleagues completed post-intervention questionnaires on behalf of research participants:

With our surveys, we're told to have them completely filled out. But you may have had a participant that did the pre-survey but not the post, and when you send it in it has to coordinate. A lot of us feel like we can't send it in incomplete, so we have to fill this out ourselves. So sometimes I fill it out myself.

Another form of fabrication of data was knowingly enrolling and interviewing ineligible persons. An example was given by a single-role CRW, who said he had coached ineligible participants on how to get into a drug treatment intervention trial where eligibility criteria included having used drugs in the last thirty days:

Now I've run into situations where they said, "I ain't used in like a month and a half." And I'll say to them, "Listen, if you really desire this help you need to say you've used in the last thirty days."

A smaller number of dual-role and single-role CRWs said they were aware of instances where a colleague 
fabricated entire interviews and study participants. One dual-role CRW gave the example of a friend, whose work involved conducting workshops to increase knowledge about sexual health and to collect pre-and post-tests of knowledge and attitudes. She talked about how some research workers dealt with the difficulties of meeting recruitment targets, saying:

I know somebody that was in that situation and it's sad to say he had to lie and make up stuff, make up people. He went into the phone book and acted like he gave a workshop to this and this person, gave the tests to this and this person.

Beyond falsification and fabrication of data, about one-third of CRWs said they or a colleague had circumvented aspects of human subjects protection concerning confidentiality and voluntariness in order to meet recruitment goals. One single-role CRW who worked on short contracts for a research agency, which required her to meet a quota in order to keep the contract, talked about how she would get a reluctant person to participate in a survey:

You tell someone that you're about to lose your job they're more sympathetic and they let you in. I do it all the time. I sit back and tell 'em, listen if I don't get this study, they gonna drop me and get somebody else. That works better than offerin' 'em money sometimes.

A different single-role CRW talked about how he and his colleague skirted participant confidentiality in order to obtain follow-up data:

We kind of go in through the back door. That may be unethical. We will call the mothers, the girlfriends, babies' mothers, whoever. And we normally don't tell them about the study because it is a confidential one, but we say, you know, we have money for them. And five minutes later that person is on the phone calling you back.

An important theme emerged in the form of stories told by CRWs about instances where they crossed usual and customary boundaries (e.g., of their job description, of their relationship with a research subject). Many of these narratives constituted violations of the research protocol and clearly posed a challenge to maintaining data integrity. For example, a dual-role CRW who worked on a drug intervention study told a story about purchasing drugs for a potential study subject who was going through withdrawal and could not be screened for study participation until a few days later. Reflecting on his actions, he said:
It was just one of them moments in your life that you just gotta make a call and even though ethically it was wrong, human-being-ly it was the thing to do for me.

About half of the CRWs told stories about actions such as giving out personal phone numbers to participants, testifying in court on behalf of a research participant who was charged with assault, and staying out late into the night with a young, pregnant research participant who had been locked out of her house by her alcoholic parent and had nowhere else to go. One striking example among many was a single-role CRW whose work involved interviewing drug-addicted mothers; when one research participant was sick and hospitalized over the holidays, the CRW took the participants' three children home with her so they would not be sent into foster care. She reflected on her actions, saying:

They had nowhere else to go. It was a choice, I guess, it was more like in the human capacity. It didn't interfere with the interviewing or nothing,

because they were only with me like three days.

While many traditional research assistants expressed caring and concern for individuals they encountered while doing their research work, not one of them said they had exceeded the boundaries of their job descriptions to provide rides, meals, or other assistance to potential or enrolled study participants.

\section{Context of Misconduct and Misbehavior by Front-Line Research Personnel}

Nearly all of the front-line research personnel we interviewed for the study, including the traditional research assistants, were dedicated to the communities they worked in and invested in the idea that the studies they worked on would result in tangible benefits to both individual participants and the community at large. How, then, are we to understand the actions taken by many of the CRWs in our sample that posed threats to the integrity of individual research studies and to the overall research endeavor? Our interviews revealed important clues about the pressures faced by research workers. In particular, we uncovered key elements of the demands placed on many CRWs, which help elucidate the context in which some CRWs commit ethical violations in the course of doing front-line research work.

Throughout the interviews, research personnel identified what they saw as the top reasons why a research worker (themselves or a colleague) might commit an ethical violation in research. The top two reasons cited by both CRWs and traditional research assistants were "pressure to meet recruitment goals" and "desire to help a 
research participant." The first reason, "pressure to meet recruitment goals," was brought up by every one of the 15 CRWs who said they had committed an ethical violation. These CRWs believed they were in danger of losing their jobs if they did not meet recruitment goals. For CRWs who were employed on a specific program or study, there was concern that the project would lose its funding. A typical comment comes from a CRW who talked about the pressures on him and other front-line research personnel when there is a discrepancy between target recruitment numbers and what can be realistically accomplished, saying:

The funders or whoever the top people are think it's so easy, but when you actually get into the field you see it's not that easy.... Sometimes the goals are set too high for some of these projects or programs just to get funding, and a lot of times they're not met and that's why funding is lost.

Another CRW who worked on a contract basis talked about the consequences to her livelihood if she did not reach "the quota" specified by her supervisor:

Then they don't call you back. They feel like you didn't put your best effort out.... They'll stop you and bring in another interviewer... they'll call your cases in and reassign them to somebody else.... It'd be a long time before you hear from them again.

The second reason given for fabricating or falsifying data-desire to help a research participant-was a bit more unexpected, especially if the research workers' primary role was to screen community members for a research study and not themselves provide direct services to potential research participants. For example, a singlerole CRW who screened community members for a drug use intervention study said he thought it was acceptable to "coach" someone who was interested in getting off drugs to help them get into the study:

Because sometimes they really want some services. They need some services.... A lot of times being caught up in the game you're not aware of the services that is out there that can help you, right? So, like if I see somebody or feel like they really want this [to get off drugs] and they're at the end of their road, I'm gonna help them.

In addition to explicit reasons given by participants about why research workers might commit ethical violations, our interviews also demonstrated how important it is to look at the context (e.g., work, physical, and social environments) in which research misconduct occurs. A number of dual-role CRWs identified primarily with their service role within the community and viewed research- related activities as an unwelcome add-on to their job duties. Some CRWs in this position may feel less inhibited when it comes to violating research ethics. For example, one dual-role CRW spoke openly about how she enrolled ineligible clients, personal friends, and family members into research to meet her recruitment goals and pass on study compensation to people she thought could benefit. She talked about fabricating responses on research instruments:

When you just fill out the paperwork in the car and nobody's there. You just take some guesses ... they don't seem like they ever been in jail, they don't seem like they ever done intravenous drugs ... I'm not gonna lie to you, I have done this.... And sometimes, like I said, we've had to, you know, maybe just use somebody just came in and not really a full-fledged client... "Oh, since you here, let me ask you these questions and you can help out with this little research," and not really see if they even qualify for the research.... It may not be what the people (researchers) are lookin' for...

Many CRWs differed from traditional research assistants in that they often identified with or said they understood and "knew" the lives of the people from whom they collected research data. This, in turn, appeared to affect their views of the interview questions, including whether questions presented a burden to research participants and whether questions were likely to yield sound data. Twenty of 32 CRW s expressed concerns that questions on research survey instruments were burdensome to community members; in contrast, this concern was expressed by only 3 of the 14 traditional research assistants.

For example, one dual-role CRW collected information on HIV risk in community settings where potential respondents included new mothers. She said she was uncomfortable asking some questions:

You come with this little packet with all these personal [questions]... asking them "Were you born as a male or female?" I mean, to me, that question... I mean she just had a baby... now, unless something has changed, you know... what else? Oh, about their partner, have their partners been in jail, did they ever have sex with somebody they met on the Internet. So it's just a lot of really personal information that they want us to get... [I feel] uncomfortable about it 'cause pretty much I would figure if somebody's askin' me those kind of questions I may think, "Well, why would you think I'm that kind of person?"

A number of CRWs were critical of survey instruments and specific questions, as in the example above, and 
skeptical of the quality of the data they gathered. For example, several dual-role CRWs observed that women receiving state or federal aid were unlikely to give honest answers to questions about income, sources of support, and marital status due to concerns about the possibility of losing benefits.

Finally, more CRWs (20 of 32) talked about dangers they faced to comfort and safety while doing research work compared to the traditional research assistants ( 3 of 14), and many CRWs experienced a lack of support from supervisors and principal investigators. A typical description of this came from a single-role CRW who conducted home-based interviews, usually on her own. Speaking of a time when a male interviewee had made advances toward her, she said she had responded by telling him she'd have to come back to interview him another day. The interviewer asked if she had reported the incident to her supervisor, and the woman responded:

I don't tell them anything. All the supervisors want to know is if you got the interview. They don't care what happens in the street or if they do, they don't ask have you had any adverse interviews. No one has asked that in the ten years that I've been out... they tell you in your training be careful, protect yourself, but afterwards no one's asking, "Is everything going okay?"

Several CRWs said they sometimes filled out interviews on behalf of a participant rather than asking the questions during a home visit due to unsanitary or unsafe conditions in the home. Other CRWs told stories about facing physical danger when conducting research activities in certain neighborhoods (e.g., being shot at, encountering drug dealers inside and outside homes). One single-role community research worker told of the time she was caught in a shoot-out while conducting door-to-door interviews in a dangerous neighborhood:

I did this study and I was in a bad neighborhood and there was a gunfight and what happened was the traffic was like locked... and there was a car behind me, so I couldn't back out. All I could do was duck and hope the bullets don't hit me. I told my manager; she said, "Okay, did you get any studies?"

In contrast, the few traditional research assistants who mentioned safety issues did so in the context of talking about safeguards in place as part of the research protocol. For example, one traditional research assistant who conducted interviews with methadone users in a communitybased methadone clinic discussed occasions when a research participant had revealed suicidal thoughts:
I've never been alone with someone when they expressed something like that. It's always been one or more of us are there, so, you know, basically what happens is as soon as any of us hear something like this, one of us is on the phone to our supervisor.... One or two of us stays with the person, somebody goes and gets the counselor. So we kind of all just do it as a team.

More CRWs told stories of risk and danger, and these narratives recounted higher levels of physical and psychological risk compared with those told by traditional research workers.

\section{Differences between Single-Role and Dual-Role CRWs}

Our interviews revealed differences between dual-role and single-role CRWs in terms of the types of misbehaviors they said they had engaged in while conducting research activities. For example, 7 out of 15 dual-role CRWs said they had fabricated responses from a research participant, while none of the 17 single-role CRWs said they had done this. A number of dual-role CRWs talked about the challenge of having research activities added to their already full plate of education and service-provision. One woman described her reaction when the organization she worked for partnered with a researcher, resulting in extra work for her and her colleagues:

Well, I don't see where they're helping me, 'cause my salary didn't change none.... They're not giving me no turkey around Thanksgiving.... It's like "so and so with the $\mathrm{PhD}$ is conducting this research" and we'll

be looking at each other and rolling our eyes because we know what comes next... more work for us.

This dual-role CRW went on to talk about how she and some of her co-workers felt justified in cutting corners when it came to collecting research data that had been added to their regular duties, saying:

You know, once you turn in that report you don't want them (supervisors) callin' and sayin' "Well, you didn't get this and you didn't get that." So, you just go ahead and make somethin' up sometimes.

In addition, dual-role CRWs typically collected research data from persons with whom they already had a service-oriented relationship; thus, in the face of time constraints, some dual-role CRWs said they prioritized the service-related aspect of their work over the researchrelated paperwork. One example comes from a dual-role community research worker who primarily provided services and education to parents of elementary schoolaged children. Talking about how she later became 
responsible for administering pre-and post-test measures to participants to evaluate the impact of the program, she said it was hard to balance the different roles. When she found that many parents left the educational sessions without completing the post-test measures, she said she had filled them out on her own. In talking about why she felt it necessary to do this, the dual-role CRW said:

We were told that this information could not be incomplete... we were told that they (the questionnaires) would be checked and this is about your job performance, and of course nobody wants to look bad and be given a bad evaluation, so you do what you have to do, or you feel that you have to do.

More of the single-role CRWs ( 9 of 15) said they had circumvented aspects of human subjects protection in order to meet recruitment goals compared to dual-role CRWs (2 of 15). Examples of this type of misconduct and misbehavior include: glossing over or omitting parts of the consent form; pressuring an individual to consent by telling him the research worker would lose his job if he didn't meet recruitment goals; and using other means to persuade an individual to participate or continue in a research study. A single-role CRW said he and his co-workers, who were responsible for evaluation of a school-based educational program, were concerned that too many teens were dropping out:

So, I know it wasn't right, but we told 'em, "Listen, your parents and teachers are going to be real disappointed in you if you don't come to the next meeting and see this [study] through."

In the case of single-role CRWs like this man, their primary role is to recruit and enroll participants in a study and to obtain follow-up data; the pressure to meet recruitment goals may lead some of them to engage in coercion of research subjects.

\section{Connection to the Research Process as a Buffer Against Pressures of Community-based Research}

Nearly all of the front-line research personnel said they faced the pressure to reach recruitment goals and other stressors described above; however, more than half of respondents said they would not fabricate or falsify data. Our analysis uncovered potential protective factors that may buffer front-line workers from the demands of recruitment and data collection in community-based settings. Specifically, we found that the traditional research assistants and CRWs who said they would not commit an ethical violation also talked about various ways in which they were "connected" to the research process and/or team. These included: being well-integrated into the research team (e.g., having an office co-located with other members of the research team, attending regular research team meetings); possessing a good understanding of the research protocol (e.g., being aware of the rationale behind inclusion/exclusion criteria or why certain measures were used to evaluate a program); having access to a supervisor or PI in case of problems (e.g., having been given a cellphone to use during home visits); and feeling respected and supported by one's supervisor. A traditional research assistant explained why he would not enroll a person who exceeded the age cut-off for a study even if it meant he fell short of recruitment goals:

Well, there are good reasons why you have to have a cut-off... you might be testing whether a drug works on a certain population and the drug might be risky for older people or something like that. There are reasons...you don't want to cut corners and take a risk that someone will get hurt or the data will be messed up.

In another example, a dual-role CRW described why she would not fabricate data in order to avoid having a research participant dropped from a study, saying that the consequences of doing so included "skew[ed] results or not making that person [research participant]'s efforts valid."

When front-line research personnel who were wellconnected to the research process and team encountered a problem while recruiting participants or collecting data, they went to a supervisor or study PI for help rather than trying to resolve it themselves. A typical example comes from a single-role CRW who discussed a time when she was not sure whether an individual was eligible to participate in the study; she asked for guidance from her direct supervisor and the study PI. She went on to characterize her relationship with her bosses, saying:

I'm very comfortable with the people that I work with and work for...my supervisor, and the person in charge of the study. I feel comfortable enough to go up to anybody's office and say I need help or ask any questions.... I'm gonna get an answer, and if they don't know, they'll find out.

Many front-line research workers who had access and proximity to higher-ups said they knew what to do when faced with an ethical problem because they had heard similar situations discussed in a team meeting or in spontaneous discussions with other study staff with nearby offices.

In contrast, CRWs who said they had committed ethical violations in response to pressure to meet recruitment 
goals and other stressors also appeared to lack a connection to the research process or team. As we discussed above, some CRWs had research activities added to their regular duties when the organization where they worked contracted with an outside investigator, while others worked on a contract basis for a researcher or research organization. These workers had no access to the study PI (and in fact, often did not even know the name of the PI) and had little or no understanding of the research protocol. Some of these CRWs questioned the motivations and intentions of researchers and expressed doubt that the research would ever benefit the communities where it was conducted. A dual-role community research worker who said she filled in survey responses herself when she was pressed for time said:

I really get to the point sometimes I don't really care, I just hand in whatever... and it's just like they [researchers] not gonna do nothin' anyway. I feel like they don't really care.... They're just tryin' to meet their quota and that way they can say they did it.

\section{Discussion}

We found the very factors that can contribute to CRWs having greater access to and potentially greater success with recruitment and data collection in some communities-shared experiences, ethnic identity, geography, vernacular and (sometimes) shared acquaintancesalso contributed to the creation of a context in which research misconduct and normal misbehaviors were more likely to occur. Despite (or in some cases, because of) their sincere desire to help the communities in which they worked and to see community members benefit from the research being done, nearly half of the CRWs said they had engaged in misconduct that posed a threat to data integrity. Many CRWs, including those who said they had engaged in misconduct and misbehaviors, questioned the overall accuracy and usefulness of findings generated from some research being done in their communities.

CRWs were more likely to say they had committed an ethical violation in research as compared to traditional research assistants; however, a limitation of our study is that behaviors were self-reported, not observed. It is possible that traditional research assistants did not disclose misconduct due to social desirability response bias. We find it more likely that traditional research assistants, because of their advanced knowledge of the research protocol and greater access to higher-level research staff, were better positioned than many CRWs to take advantage of "gray areas" in research and to explore alternative options.
Thus, when faced with difficulties related to recruitment and follow-up, rather than fabricating data or manipulating potential participants as some CRWs did, traditional research assistants were able to refer a difficult-to-reach participant to another person on the team described as expert at recruitment. Additionally, traditional research assistants were younger, more likely to have a college education or graduate degree, and had worked fewer years in their research jobs compared with CRWs. Thus, traditional research assistants may respond to threats to safety and comfort encountered when conducting research in community settings by reporting problems to a supervisor or moving onto another job, while many CRWs may feel "trapped" in their job and respond by fabricating data to avoid going into a potentially unsafe home.

Our findings indicate that being more connected to the research process and/or research team-having specific knowledge about the research protocol, believing that the research (as it was designed) was likely to benefit the community, and having access to colleagues and supervisors-acted as a buffering factor for front-line research staff who did not engage in ethical misconduct, despite facing the same pressures to meet recruitment goals and other stressors of conducting communitybased research. Many CRWs said they would not enroll an ineligible person or fabricate or falsify data because of concern for the integrity of the study. A number of front-line research personnel cited mechanisms at their organization for detecting such actions as one reason for not engaging in ethical misconduct.

Some researchers have written about the potential for front-line community health workers to contribute to research and to serve as advocates for social justice (Perez $\&$ Martinez, 2008). We agree with the notion that these front-line workers can cast a critical eye on the research being done in their communities. We heard from a number of CRWs who "turned the lens" back onto grant makers and investigators who exerted control over the research agenda. In some instances, CRWs questioned whether methods and instruments would yield an accurate picture of a phenomenon or community, while others expressed concern that some research in minority communities, while necessary and important, might result in stigmatization of those communities.

In contrast to the depiction of front-line workers as potential advocates and policymakers in some of the literature (Flax \& Earp, 1999; Lewin et al., 2005; Perez \& Martinez, 2008; Popay \& Williams, 1996), many of the stories we heard raised concerns about the social justice implications of using CRWs to increase research participation in under-represented communities and enhance investigators' ability to meet recruitment goals. Many of 
the CRWs were as vulnerable as the people with whom they were conducting research and/or providing services. CRWs were more likely to be exposed to physical danger than were traditional research assistants and were less likely to have recourse to other work if they lost their jobs due to lack of funding. In research ethics, justice means that benefits and burdens of research are spread equally, and our interviews revealed that the burdens of conducting front-line research weighed more heavily on CRWs. Despite facing great stressors and (in some cases) threats to safety, the CRWs we interviewed were committed to bettering the lives of people in their communities. We hope that our findings will spur improvements to research design and structures of support for these frontline research personnel, thereby enhancing not only the integrity of studies on which they work but also the conditions under which they do their work.

\section{Research Agenda}

More research is needed to fully understand the consequences, both positive and negative, of the growing reliance on community members as recruiters and data gatherers in health services and translational research (Simon \& Mosavel, 2010). Since our qualitative research study was limited to a small number of CRWs within a specific region of the country and is therefore limited in generalizability, more systematic study of the experiences and behaviors of CRWs is necessary. We also need to compare the effectiveness of CRWs and traditional research workers employed on the same study, something that has rarely been done (Clark et al., 1999; Holbrook, Farrar, \& Popkin, 2006).

\section{Best Practices and Educational Implications}

Our study indicates that standard training in protection of human subjects is not sufficient to meet the demands put upon CRWs conducting front-line research work. Any training developed for these research personnel must not only take into account learning styles, language, culture, and literacy issues, but must also address the real-world stresses and pressures faced by CRWs. These trainings should emphasize how to make decisions "on the ground" over rote memorization of ethical guidelines and should feature scenarios that reflect the realities of conducting research in marginalized neighborhoods. Most importantly, organizations and studies that employ CRWs should make efforts to promote connection to the research; access to supervisors and colleagues should be ongoing, close, and collaborative. In addition, research personnel should receive training that includes some explanation of the rationale behind aspects of the protocol (e.g., inclusion/ exclusion criteria) and the consequences of misconduct to the study and the larger research endeavor. Finally, PIs and other higher-level research personnel should endeavor to understand the realities encountered by front-line research personnel; for example, by visiting the field or holding regular "debriefing" meetings with these research staff.

Overall, the traditional research assistants were more easily able to "enter and leave" the worlds of the communities where they conducted research. In contrast, CRWs were more likely to conduct home visits with research participants, more likely to live in the same neighborhoods, and more likely to say they identified to some extent with their research participants. These factors made it difficult for many CRWs to maintain boundaries between self and work; such boundaries can be key to protecting the safety and mental health of front-line research personnel and also to maintaining the integrity of research data. CRWs would benefit from opportunities for continuing education and training in research protocols and ethical issues and problems faced "on the ground" in doing their work, as well as the development of continuing education events that put them together with research personnel from other cities and/or community-placed studies.

\section{Acknowledgments}

This research was supported by 1RO1NR009879 to Leslie Alexander. We thank Kay Armstrong, Patrice Gammon, Diana Harris, Page Buck, and Taalibah Kariem-White for conducting study interviews and providing other assistance with the research. We are indebted to the organizations and investigators who facilitated our recruitment efforts, and especially to the front-line research personnel who so generously shared their experiences and perspectives with us. The reviewers' comments on an earlier draft of this manuscript are greatly appreciated. Disclaimer: The contents of this article do not represent the views of the Department of Veterans Affairs or the United States Government.

\section{Author Note}

Address correspondence to: Gala True, Center for Health Equity Research and Promotion, Philadelphia VA Medical Center, 9E, 3900 Woodland Avenue, Philadelphia, PA 19104. Phone: 215-823-5800, ext. 5780; E-MAIL: Jennifer.True2@va.gov. 


\section{Authors' Biographical Sketches}

Gala True is Core Investigator at the Center for Health Equity Research and Promotion at the Philadelphia Veterans Affairs Medical Center, where her research focuses on patient-centered approaches to understanding and reducing health disparities and access to care. She collaborated on research planning, instrument development, and data collection, and led the team on qualitative data analysis on this study.
Leslie Alexander is Professor of Social Work and Social Research and Chair of the IRB at Bryn Mawr College. She led and was involved in every phase of the project reported here.

Kenneth A. Richman is Professor of Philosophy and Health Care Ethics at the Massachusetts College of Pharmacy and Health Sciences, where he also serves as IRB Chair. He was involved in research planning, instrument design and revision, and data analysis for the study reported here.

\section{References}

Alexander, L. \& Richman, K. (2008). Ethical dilemmas in evaluations using indigenous research workers. American Journal of Evaluation, 29(1), 73-85.

Anderson, M. S., Horn, A. S., Risbey, K. R., Ronning, E. A., De VRies, R., \& Martinson, B. C. (2007). What do mentoring and training in the responsible conduct of research have to do with scientists' misbehavior? Findings from a National Survey of NIH-funded scientists. Academic Medicine, 82(9), 853-860.

Anderson, M. S., Martinson, B. C., \& De Vries, R. (2007). Normative dissonance in science: Results from a national survey of U.S. scientists. Journal of Empirical Research on Human Research Ethics, 2(4), 3-14.

Anderson, M. S., Ronning, E. A., De Vries, R., \& Martinson, B. C. (2007). The perverse effects of competition on scientists' work and relationships. Science and Engineering Ethics, 13(4), 437-461.

BRAND, H. R. (2002). Interviewing: Unstructured and semistructured. In Research Methods in Anthropology (3rd ed., pp. 203-239). Walnut Creek, CA: AltaMira Press.

Clark, C. C., Scott, E. A., Boydell, K. M., \& Goering, P. (1999). Effects of client interviewers on client-reported satisfaction with mental health services. Psychiatric Services, 50(7), 961-963.

de Vries, R., Anderson, M. S., \& Martinson, B. C. (2006). Normal misbehavior: Scientists talk about the ethics of research. Journal of Empirical Research on Human Research Ethics, 1(1), 43-50.

FLAX, V. L. \& EARP, J. L. (1999). Counseled women's perspectives on their interactions with lay health advisors: A feasibility study. Health Education Research, 14(1), 15-24.
Hill, M. N., Bone, L. R., \& Butz, A. M. (1996). Enhancing the role of community-health workers in research. Image-The Journal of Nursing Scholarship, 28(3), 221-226.

Holbrook, A. L., Farrar, I. C., \& Popkin, S. J. (2006). Surveying a Chicago public housing development: Methodological challenges and lessons learned. Evaluation Review, 30(6), 779-802.

Lewin, S. A., Dick, J., Pond, P., Zwarenstein, M., AJA, G., VAN WYK, B. ET AL. (2005). Lay health workers in primary and community health care. Cochrane Database of Systematic Reviews (1), CD004015.

MacQueen, K. M., McLellan, E., Metzger, D. S., Kegeles, S., Strauss, R. P., Scotti, R. ET AL. (2001). What is community? An evidence-based definition for participatory public health. American Journal of Public Health, 91(12), 1929-1938.

Martinson, B. C., Anderson, M. S., Crain, A. L., \& de Vries, R. (2006). Scientists' perceptions of organizational justice and self-reported misbehaviors. Journal of Empirical Research on Human Research Ethics, 1(1), 51-66.

Martinson, B. C., Anderson, M. S., \& De Vries, R. (2005). Scientists behaving badly. Nature, 435(7043), 737-738.

Perez, L. M. \& Martinez, J. (2008). Community health workers: Social justice and policy advocates for community health and well-being. American Journal of Public Health, 98(1), $11-14$.

Popay, J. \& Williams, G. (1996). Public health research and lay knowledge. Social Science and Medicine, 42(5), 759-768.

Simon, C. \& Mosavel, M. (2010). Community members as recruiters of human subjects: Ethical considerations. American Journal of Bioethics, 10(3), 3-11. 
Reproduced with permission of the copyright owner. Further reproduction prohibited without permission. 